\title{
A Formal Proof of Cauchy's Residue Theorem
}

\author{
Wenda Li and Lawrence C. Paulson \\ Computer Laboratory, University of Cambridge \\ $\{$ w1302,1p15\}@cam.ac.uk
}

\begin{abstract}
We present a formalization of Cauchy's residue theorem and two of its corollaries: the argument principle and Rouché's theorem. These results have applications to verify algorithms in computer algebra and demonstrate Isabelle/HOL's complex analysis library.
\end{abstract}

\section{Introduction}

Cauchy's residue theorem - along with its immediate consequences, the argument principle and Rouché's theorem - are important results for reasoning about isolated singularities and zeros of holomorphic functions in complex analysis. They are described in almost every textbook in complex analysis [3, 15, 16].

Our main motivation of this formalization is to certify the standard quantifier elimination procedure for real arithmetic: cylindrical algebraic decomposition [4]. Rouché's theorem can be used to verify a key step of this procedure: Collins' projection operation [8. Moreover, Cauchy's residue theorem can be used to evaluate improper integrals like

$$
\int_{-\infty}^{\infty} \frac{e^{i t z}}{z^{2}+1} d z=\pi e^{-|t|}
$$

Our main contribution ${ }^{1}$ is two-fold:

- Our machine-assisted formalization of Cauchy's residue theorem and two of its corollaries is new, as far as we know.

- This paper also illustrates the second author's achievement of porting major analytic results, such as Cauchy's integral theorem and Cauchy's integral formula, from HOL Light [12].

The paper begins with some background on complex analysis (Sect. 2), followed by a proof of the residue theorem, then the argument principle and Rouché's theorem (3-5). Then there is a brief discussion of related work (Sect. 6) followed by conclusions (Sect. 7).

\section{Background}

We briefly introduce some basic complex analysis from Isabelle/HOL's Multivariate Analysis library. Most of the material in this section was first formalized in HOL Light by John Harrison [12] and later ported to Isabelle.

\footnotetext{
${ }^{1}$ Source is available from https://bitbucket.org/liwenda1990/src_itp_2016/src
} 


\subsection{Contour Integrals}

Given a path $\gamma$, a map from the real interval $[0,1]$ to $\mathbb{C}$, the contour integral of a complex-valued function $f$ on $\gamma$ can be defined as

$$
\oint_{\gamma} f=\int_{0}^{1} f(\gamma(t)) \gamma^{\prime}(t) d t
$$

Because integrals do not always exist, this notion is formalised as a relation:

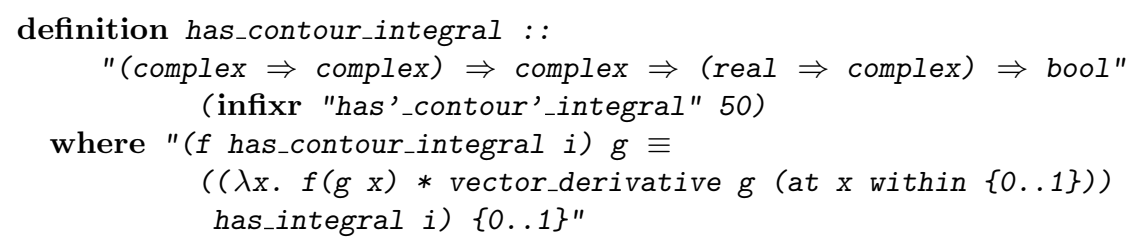

We can introduce an operator for the integral to use in situations when we know that the integral exists. This is analogous to the treatment of ordinary integrals, derivatives, etc., in HOL Light [12] as well as Isabelle/HOL.

\subsection{Valid Path}

In order to guarantee the existence of the contour integral, we need to place some restrictions on paths. A valid path is a piecewise continuously differentiable function on [0..1]. In plain English, the function must have a derivative on all but finitely many points, and this derivative must also be continuous.

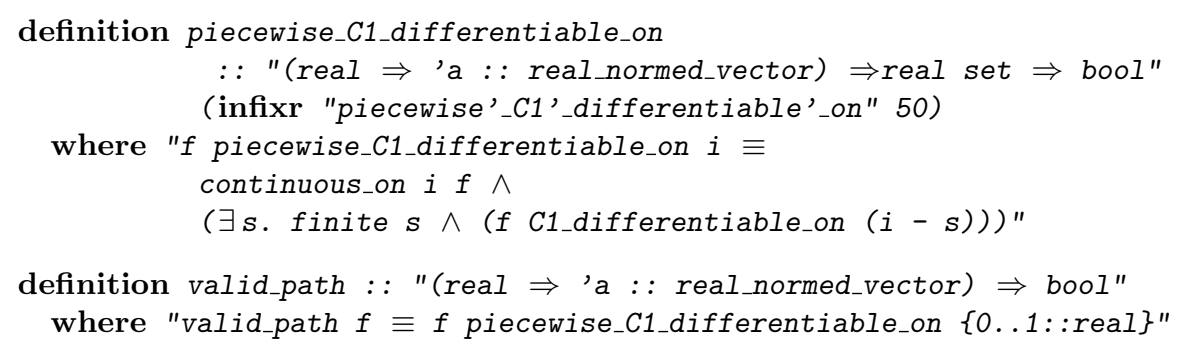

\subsection{Winding Number}

The winding number of the path $\gamma$ at the point $z$ is defined (following textbook definitions) as

$$
n(\gamma, z)=\frac{1}{2 \pi i} \oint_{\gamma} \frac{d w}{w-z}
$$

A lemma to illustrate this definition is as follows:

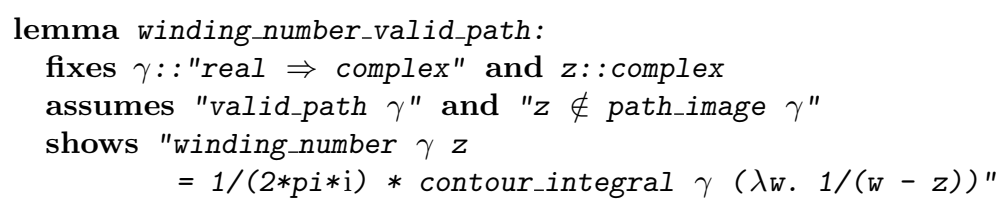




\subsection{Holomorphic Functions and Cauchy's Integral Theorem}

A function is holomorphic if it is complex differentiable in a neighborhood of every point in its domain. The Isabelle/HOL version follows that of HOL Light:

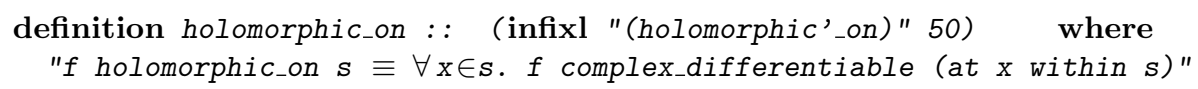

As a starting point to reason about holomorphic functions, it is fortunate that John Harrison has made the effort to prove Cauchy's integral theorem in a rather general form:

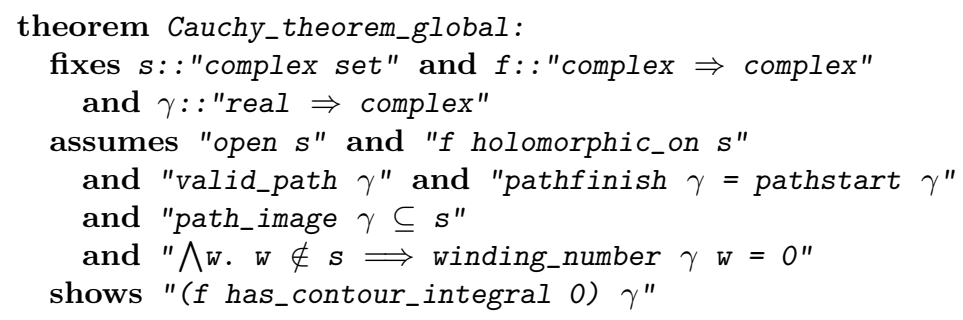

Note, a more common statement of Cauchy's integral theorem requires the open set $s$ to be simply connected (connected and without holes). Here, the simple connectedness is encoded by a homologous assumption

" $\$ w. $w \notin s \Longrightarrow$ winding_number $\gamma w=0 "$

The reason behind this homologous assumption is that a non-simply-connected set $s$ should contain a cycle $\gamma$ and a point $a$ within one of its holes, such that winding_number $\gamma$ a is non-zero. Statements of such homologous version of Cauchy's integral theorem can be found in standard texts[1, 15].

\subsection{Remarks on the Porting Efforts}

We have been translating the HOL Light proofs manually in order to make them more general and more legible. In the HOL Light library, all theorems are proved for $\mathbb{R}^{n}$, where $\mathrm{n}$ is a positive integer encoded as a type [14. The type of complex numbers is identified with $\mathbb{R}^{2}$, and sometimes the type of real numbers must be coded as $\mathbb{R}^{1}$. Even worse, the ordered pair $(\mathrm{x}, \mathrm{y})$ must be coded, using complicated translations, as $\mathbb{R}^{m+n}$. We are able to eliminate virtually all mention of $\mathbb{R}^{n}$ in favour of more abstract notions such as topological or metric spaces. Moreover, our library consists of legible structured proofs, where the formal development is evident from the proof script alone.

\section{Cauchy's Residue Theorem}

As a result of Cauchy's integral theorem, if $f$ is a holomorphic function on a simply connected open set $s$ which contains a closed path $\gamma$, then

$$
\oint_{\gamma} f(w)=0
$$


However, if the set $s$ does have a hole, then Cauchy's integral theorem will not apply. For example, consider $f(w)=\frac{1}{w}$ so that $f$ has a pole at $w=0$, and $\gamma$ is the circle path $\gamma(t)=e^{2 \pi i t}$ :

$$
\oint_{\gamma} \frac{d w}{w}=\int_{0}^{1} \frac{1}{e^{2 \pi i t}}\left(\frac{d}{d t} e^{2 \pi i t}\right) d t=\int_{0}^{1} 2 \pi i d t=2 \pi i \neq 0
$$

Cauchy's residue theorem applies when a function is holomorphic on an open set except for a finite number of points (i.e. isolated singularities):

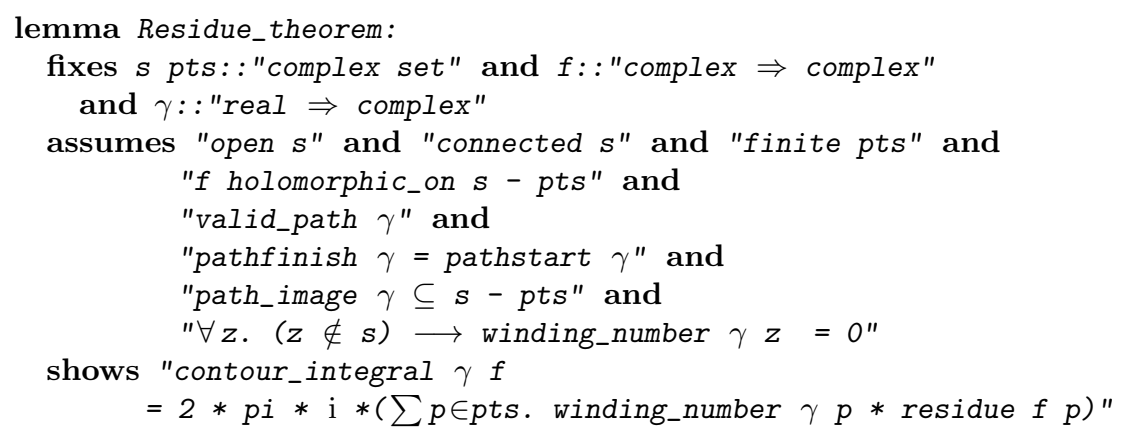

where residue $f p$ denotes the residue of $f$ at $p$, which we will describe in details in the next subsection.

Note, definitions and lemmas described from this section onwards are our original proofs (i.e. not ported from HOL Light) except where clearly noted.

\subsection{Residue}

A complex function $f$ is defined to have an isolated singularity at point $z$, if $f$ is holomorphic on an open disc centered at $z$ but not at $z$.

We now define residue $f z$ to be the path integral of $f$ (divided by a constant $2 \pi i$ ) along a small circle path around $z$ :

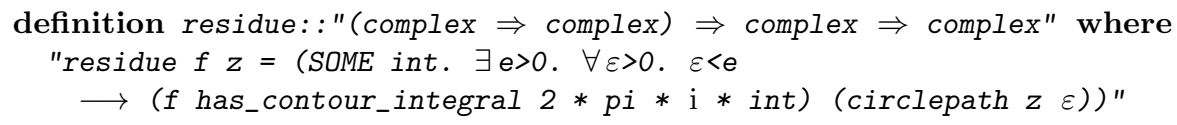

To actually utilize our definition, we need not only to show the existence of such integral but also its invariance when the radius of the circle path becomes sufficiently small.

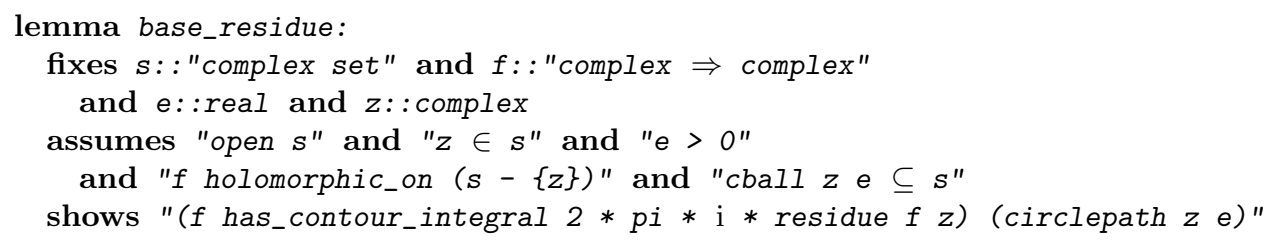

Here cball denotes the familiar concept of a closed ball: 


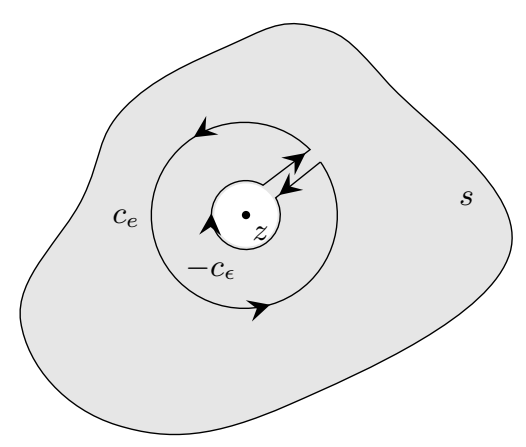

Fig. 1. Circlepath $c_{e}$ and $c_{\epsilon}$ around an isolated singularity $z$

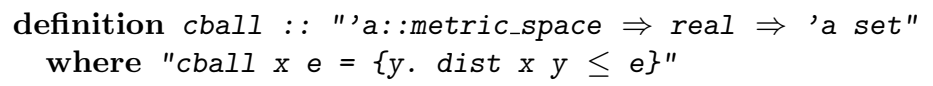

Proof. Given two small circle path $c_{\epsilon}$ and $c_{e}$ around $z$ with radius $\epsilon$ and $e$ respectively, we want to show that

$$
\oint_{c_{\epsilon}} f=\oint_{c_{e}} f
$$

Let $\gamma$ is a line path from the end of $c_{e}$ to the start of $-c_{\epsilon}$. As illustrated in Fig. 1. consider the path

$$
\Gamma=c_{e}+\gamma+\left(-c_{\epsilon}\right)+(-\gamma)
$$

where + is path concatenation, and $-c_{\epsilon}$ and $-\gamma$ are reverse paths of $c_{\epsilon}$ and $\gamma$ respectively. As $\Gamma$ is a valid closed path and $f$ is holomorphic on the interior of $\Gamma$, we have

$$
\oint_{\Gamma} f=\oint_{c_{e}} f+\oint_{\gamma} f+\left(-\oint_{c_{\epsilon}} f\right)+\left(-\oint_{\gamma} f\right)=\oint_{c_{e}} f-\oint_{c_{\epsilon}} f=0
$$

hence

$$
\oint_{c_{\epsilon}} f=\oint_{c_{e}} f
$$

and the proof is completed.

\subsection{Generalization to a Finite Number of Singularities}

The lemma base_residue can be viewed as a special case of the lemma Residue_theorem where there is only one singularity point and $\gamma$ is a circle path. In this section, we will describe our proofs of generalizing the lemma base_residue to a plane with finite number of singularities.

First, we need the Stone-Weierstrass theorem, which approximates continuous functions on a compact set using polynomial functions 2

\footnotetext{
${ }^{2}$ Our formalization is based on a proof by Brosowski and Deutsch [7].
} 


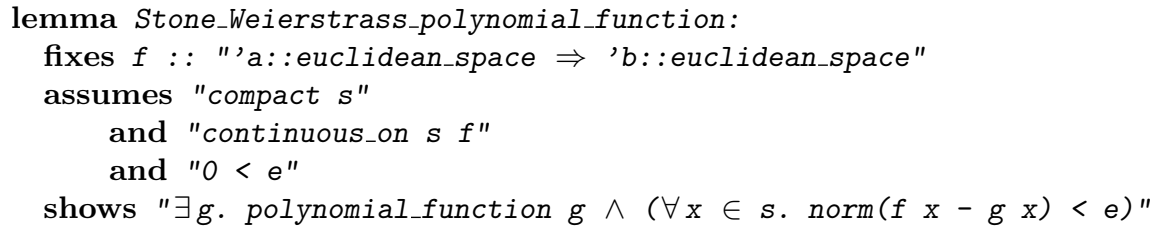

From the Stone-Weierstrass theorem, it follows that each open connected set is actually valid path connected (recall that our valid paths are piecewise continuous differentiable functions on the closed interval $[0,1])$ :

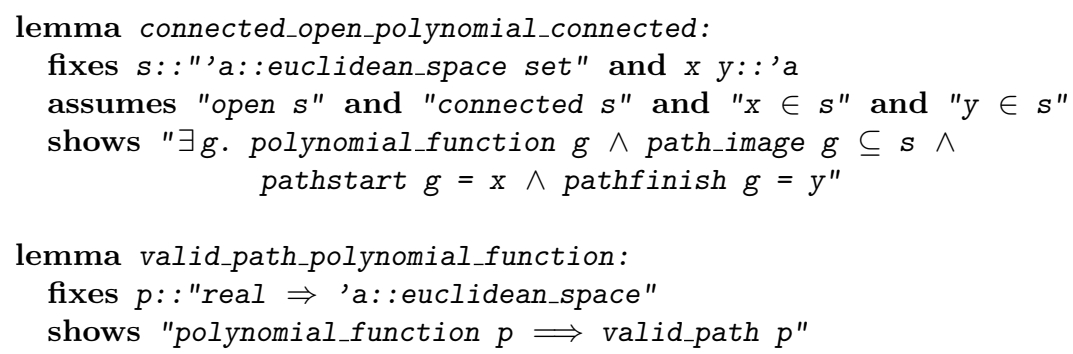

This yields a valid path $\gamma$ on some connected punctured set such that a holomorphic function has an integral along $\gamma$ :

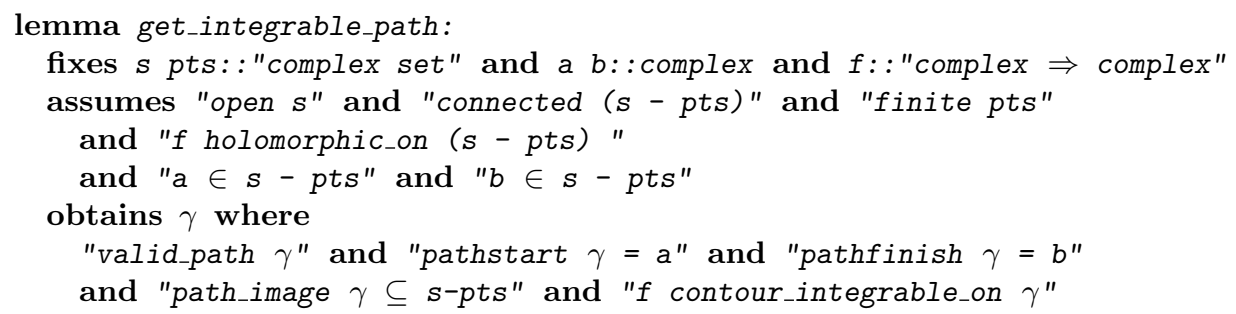

Finally, we obtain a lemma that reduces the integral along $\gamma$ to a sum of integrals over small circles around singularities:

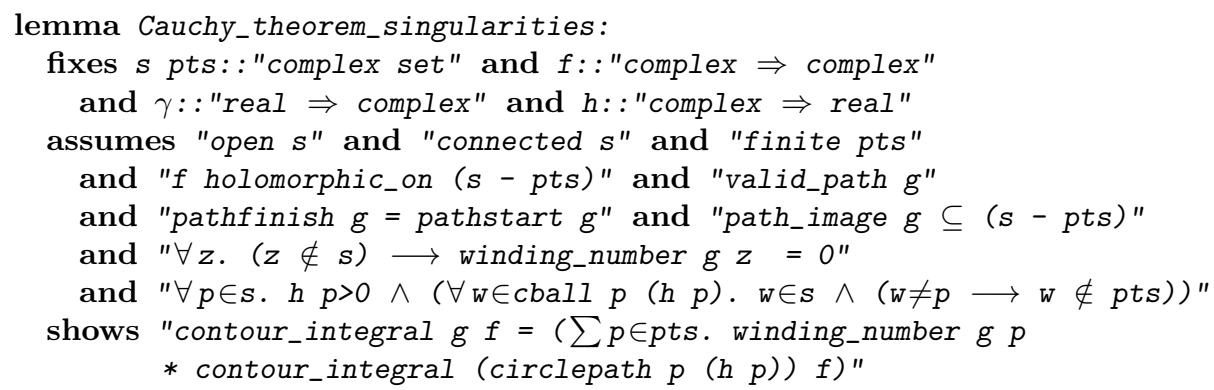

Proof. Since the number of singularities pts is finite, we do induction on them. Assuming the lemma holds when there are $p t s$ singularities, we aim to show the lemma for $\{q\} \cup p t s$. 


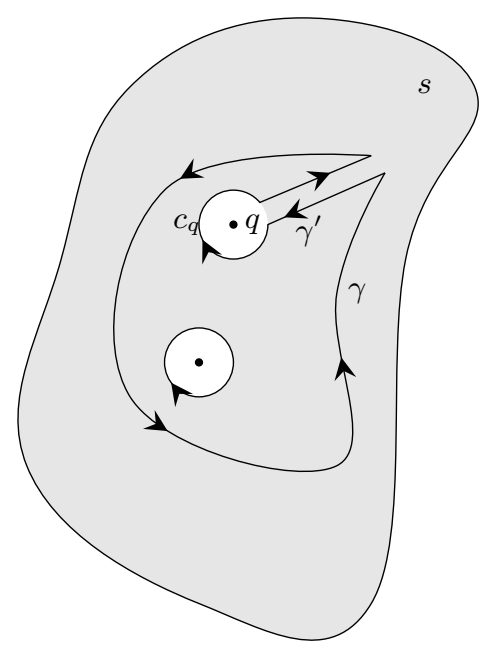

Fig. 2. Induction on the number of singularities

As illustrated in Fig. 2, suppose $c_{q}$ is a (small) circle path around $q$, by the lemma get_integrable_path, we can obtain a valid path $\gamma^{\prime}$ from the end of $\gamma$ to the start of $c_{q}$ such that $f$ has an integral along $\gamma^{\prime}$.

Consider the path

$$
\Gamma=\gamma+\gamma^{\prime}+\underbrace{c_{q}+\ldots+c_{q}}_{n(\gamma, q)}+\left(-\gamma^{\prime}\right)
$$

where + is path concatenation, $n(\gamma, q)$ is the winding number of the path $\gamma$ around $q$ and $-\gamma^{\prime}$ is the reverse path of $\gamma^{\prime}$. We can show that $\Gamma$ is a valid cycle path and the induction hypothesis applies to $\Gamma$, that is

$$
\oint_{\Gamma} f=\sum_{p \in p t s} n(\gamma, p) \oint_{c_{p}} f
$$

hence

$$
\oint_{\gamma} f+\oint_{\gamma^{\prime}} f+n(\gamma, q) \oint_{c_{q}} f-\oint_{\gamma^{\prime}} f=\sum_{p \in p t s} n(\gamma, p) \oint_{c_{p}} f
$$

and finally

$$
\oint_{\gamma} f=\sum_{p \in\{q\} \cup p t s} n(\gamma, p) \oint_{c_{p}} f
$$

which concludes the proof.

By combining the lemma Cauchy_theorem_singularities and base_residue, we can finish the proof of Cauchy's residue theorem (i.e. the lemma Residue_theorem). 


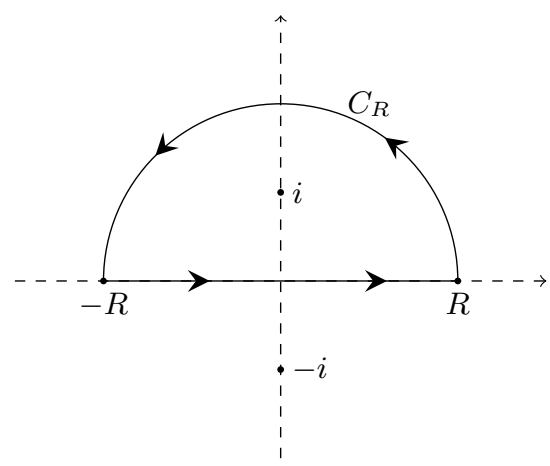

Fig. 3. A semicircle path centered at 0 with radius $R>1$

\subsection{Applications}

Besides corollaries like the argument principle and Rouché's theorem, which we will describe later, Cauchy's residue theorem is useful when evaluating improper integrals.

For example, evaluating an improper integral:

$$
\int_{-\infty}^{\infty} \frac{d x}{x^{2}+1}=\pi
$$

corresponds the following lemma in Isabelle/HOL:

lemma improper_Ex:

"Lim at_top $\left(\lambda R\right.$. integral $\left.\{-R . R\}\left(\lambda x .1 /\left(x^{2}+1\right)\right)\right)=p i "$

Proof. Let

$$
f(z)=\frac{1}{z^{2}+1}
$$

Now $f(z)$ is holomorphic on $\mathbb{C}$ except for two poles when $z=i$ or $z=-i$. We can then construct a semicircle path $\gamma_{R}+C_{R}$, where $\gamma_{R}$ is a line path from $-R$ to $R$ and $C_{R}$ is an arc from $R$ to $-R$, as illustrated in Fig. 3. From Cauchy's residue theorem, we obtain

$$
\oint_{\gamma_{R}+C_{R}} f=\operatorname{Res}(f, i)=\pi
$$

where $\operatorname{Res}(f, i)$ is the residue of $f$ at $i$. Moreover, we have

$$
\left|\oint_{C_{R}} f\right| \leq \frac{1}{R^{2}-1} \pi R
$$

as $|f(z)|$ is bounded by $1 /\left(R^{2}-1\right)$ when $z$ is on $C_{R}$ and $R$ is large enough. Hence,

$$
\oint_{C_{R}} f \rightarrow 0 \text { when } \quad R \rightarrow \infty
$$


and therefore

$$
\int_{-\infty}^{\infty} \frac{d x}{x^{2}+1}=\oint_{\gamma_{R}} f=\oint_{\gamma_{R}+C_{R}} f=\pi \quad \text { when } \quad R \rightarrow \infty
$$

which concludes the proof.

Evaluating such improper integrals was difficult for Avigad et al. 2] in their formalization of the Central Limit Theorem. We hope our development could facilitate such proofs in the future, though it may not be immediate as their proof is based on a different integration operator.

\subsection{Remarks on the Formalization}

It is surprising that we encountered difficulties when generalizing the lemma base_residue to the case of a finite number of poles. Several complex analysis textbooks [9, 16] omit proofs for this part (giving the impression that the proof is trivial). Our statement of the lemma Cauchy_theorem_singularities follows the statement of Theorem 2.4, Chapter IV of Lang [15], but we were reluctant to follow his proof of generalizing paths to chains for fear of complicating existing theories. In the end, we devised proofs for this lemma on our own with inspiration from Stein and Shakarchi's concept of a keyhole [16].

Another tricky part we have encountered is in the proof of the lemma improper_Ex. When showing

$$
\oint_{\gamma_{R}+C_{R}} f=\operatorname{Res}(f, i)=\pi
$$

it is necessary to show $i(-i)$ is inside (outside) the semicircle path $\gamma_{R}+C_{R}$, that is,

$$
n\left(i, \gamma_{R}+C_{R}\right)=1 \wedge n\left(-i, \gamma_{R}+C_{R}\right)=0
$$

where $n$ is the winding number operation. Such proof is straightforward for humans when looking at Fig. 3. However, to formally prove it in Isabelle/HOL, we ended up manually constructing some ad-hoc counter examples and employed proof by contradiction several times. Partially due to this reason, our proof of the lemma improper_Ex is around 300 lines of code, which we believe can be improved in the future.

\section{The Argument Principle}

In complex analysis, the argument principle is a lemma to describe the difference between the number of zeros and poles of a meromorphi ${ }^{3}$ function.

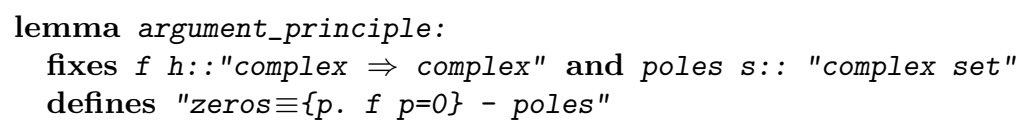

\footnotetext{
${ }^{3}$ holomorphic except for isolated poles
} 


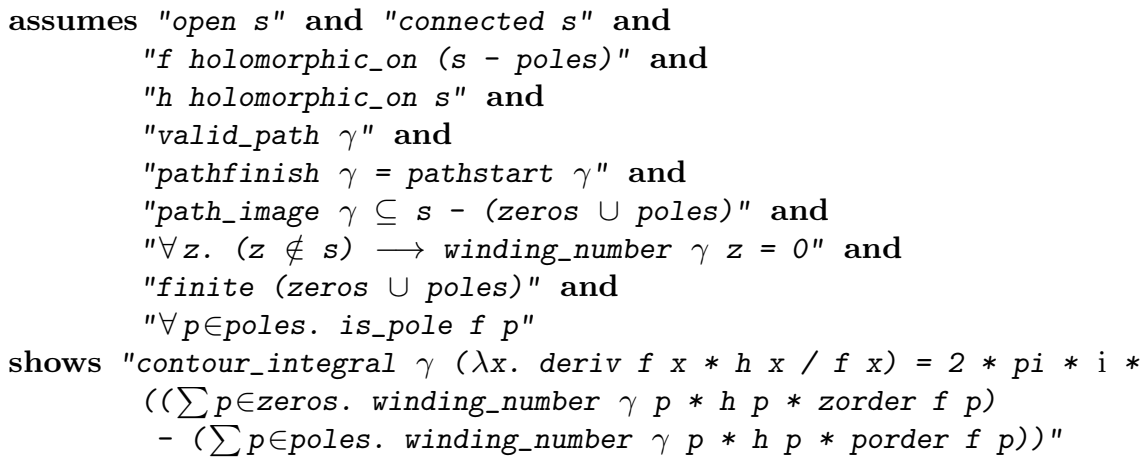

where

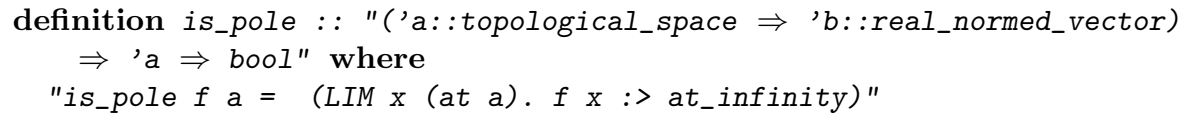

encodes the usual definition of poles (i.e. $f$ approaches infinity as $x$ approaches a). zorder and porder are the order of zeros and poles, which we will define in detail in the next subsection.

\subsection{Zeros and Poles}

A complex number $z$ is referred as a zero of a holomorphic function $f$ if $f(z)=0$. And there is a local factorization property about $f(z)$ :

lemma holomorphic_factor_zero_Ex1:

fixes s::"complex set" and $f::$ "complex $\Rightarrow$ complex" and $z:$ :complex

assumes "open $s$ " and "connected $s$ " and $z \in s "$ and $" f(z)=0 "$

and " $f$ holomorphic_on $s "$ and $" \exists \mathrm{w} \in \mathrm{s}$. $f \mathrm{w} \neq 0$ "

shows $" \exists ! n . \exists g r .0<n \wedge 0<r \wedge$ ball $z r \subseteq s \wedge$

$g$ holomorphic_on ball z $r$

$\wedge\left(\forall \mathrm{w} \in\right.$ ball $z$ r. $\left.f_{\mathrm{w}}=(\mathrm{w}-\mathrm{z})^{\wedge} n * g \mathrm{w} \wedge \mathrm{g} \mathrm{w} \neq 0\right) "$

Here a ball, as usual, is an open neighborhood centred on a given point:

definition ball :: "'a: :metric_space $\Rightarrow$ real $\Rightarrow$ 'a set"

where "ball $\mathrm{x} e=\{y$. dist $\mathrm{x} y<e\} "$

Proof. 4 As $f$ is holomorphic, $f$ has a power expansion locally around $z$ :

$$
f(w)=\sum_{k=0}^{\infty} a_{k}(w-z)^{k}
$$

\footnotetext{
${ }^{4}$ The existence proof of such $n, g$ and $r$ is ported from HOL Light, while we have shown the uniqueness of $n$ on our own.
} 
and since $f$ does not vanish identically, there exists a smallest $n$ such that $a_{n} \neq 0$. Therefore

$$
f(w)=\sum_{k=n}^{\infty} a_{k}(w-z)^{k}=(w-z)^{n} \sum_{k=0}^{\infty} a_{k+n}(w-z)^{k}=(w-z)^{n} g(w)
$$

and the function $g(w)$ is holomorphic and non-vanishing near $z$ due to $a_{n} \neq 0$.

Also, we can show that this $n$ is unique, by assuming there exist $m$ and another locally holomorphic function $h(w)$ such that

$$
f(w)=(w-z)^{n} g(w)=(w-z)^{m} h(w)
$$

and $h(w) \neq 0$. If $m>n$, then

$$
g(w)=(w-z)^{m-n} h(w)
$$

and this yields $g(w) \rightarrow 0$ when $w \rightarrow z$, which contradicts the fact that $g(w) \neq 0$. If $n>m$, then similarly $h(w) \rightarrow 0$ when $w \rightarrow z$, which contradicts $h(w) \neq 0$. Hence, $n=m$, and the proof is completed.

The unique $n$ in the lemma holomorphic_factor_zero_Ex1 is usually referred as the order/multiplicity of the zero of $f$ at $z$ :

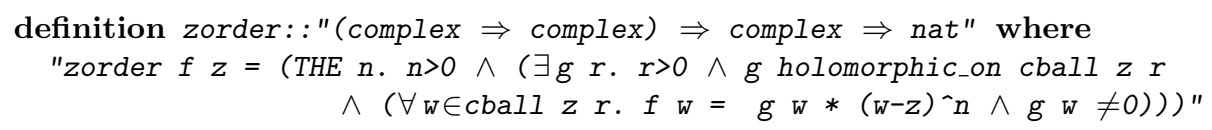

We can also refer the complex function $g$ in the lemma holomorphic_factor_zero_Ex 1 using Hilbert's epsilon operator in Isabelle/HOL:

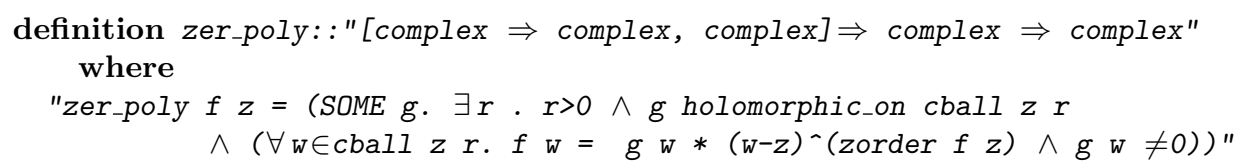

Given a complex function $f$ that has a pole at $z$ and is also holomorphic near (but not at) $z$, we know the function

$$
\lambda x \text {. if } x=z \text { then } 0 \text { else } \frac{1}{f(x)}
$$

has a zero at $z$ and is holomorphic near (and at) $z$. On the top of the definition of the order of zeros, we can define the order/multiplicity of the pole of $f$ at $z$ :

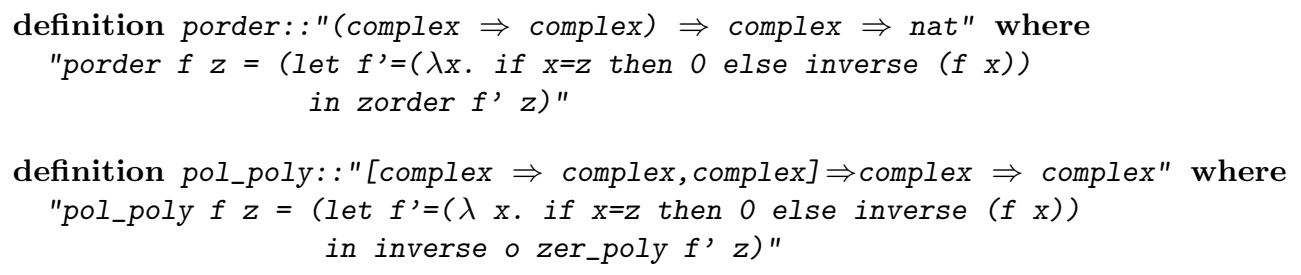


and a lemma to describe a similar relationship among $f$, porder and pol_poly:

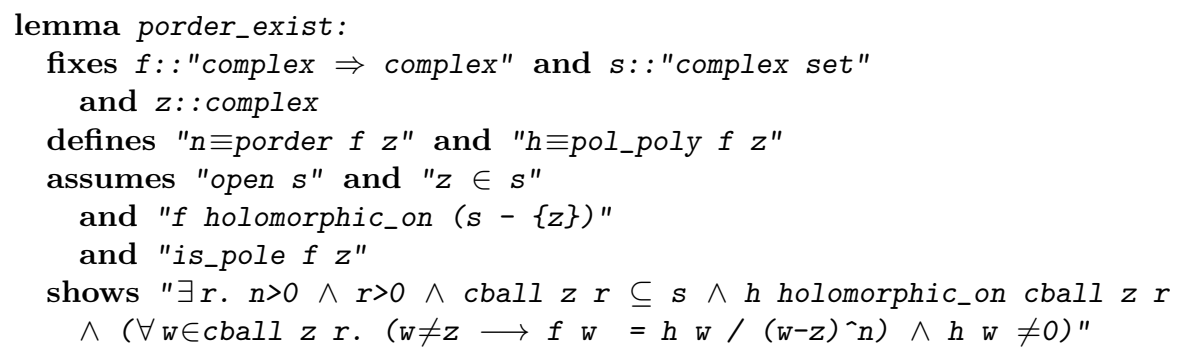

Proof. With the lemma holomorphic_factor_zero_Ex1, we derive that there exist $n$ and $g$ such that

$$
\text { if } w=z \text { then } 0 \text { else } \frac{1}{f(w)}=(w-z)^{n} g(w)
$$

and $g(w) \neq 0$ for $w$ near $z$. Hence

$$
f(w)=\frac{\frac{1}{g(w)}}{(w-z)^{n}}=\frac{h(w)}{(w-z)^{n}}
$$

when $w \neq z$. Also, $h(w) \neq 0$ due to $g(w) \neq 0$. This concludes the proof.

Moreover, porder and pol_poly can be used to construct an alternative definition of residue when the singularity is a pole.

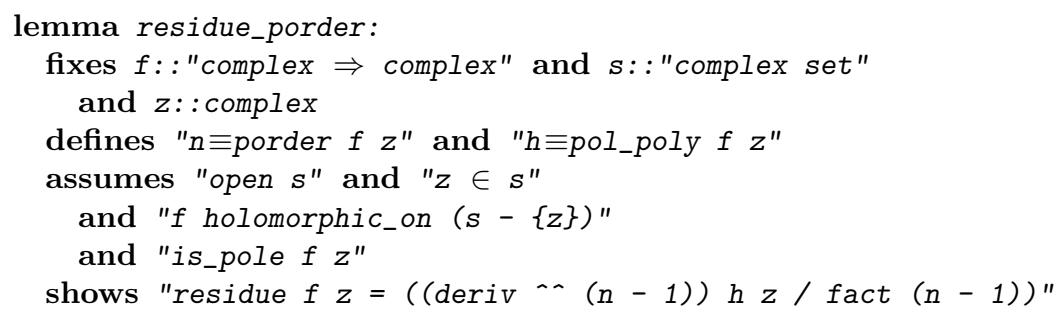

Proof. The idea behind the lemma residue_porder is to view $f(w)$ as $h(w) /(w-$ $z)^{n}$, hence the conclusion becomes

$$
\frac{1}{2 \pi i} \oint_{c_{\epsilon}} \frac{h(w)}{(w-z)^{n}} d w=\frac{1}{(n-1) !} \frac{d^{n-1}}{d w^{n-1}} h(z)
$$

which can be then solved by Cauchy's integral formula.

\subsection{The Main Proof}

The main idea behind the proof of the lemma argument_principle is to exploit the local factorization properties at zeros and poles, and then apply the Residue theorem. 
Proof (the argument principle). Suppose $f$ has a zero of order $m$ when $w=z$. Then $f(w)=(w-z)^{m} g(w)$ and $g(w) \neq 0$. Hence,

$$
\frac{f^{\prime}(w)}{f(w)}=\frac{m}{w-z}+\frac{g^{\prime}(w)}{g(w)}
$$

which leads to

$$
\oint_{\gamma} \frac{f^{\prime}(w) h(w)}{f(w)}=\oint_{\gamma} \frac{m h(w)}{w-z}=m h(z)
$$

since

$$
\lambda w \cdot \frac{g^{\prime}(w) h(w)}{g(w)}
$$

is holomorphic $\left(g, g^{\prime}\right.$ and $h$ are holomorphic and $\left.g(w) \neq 0\right)$.

Similarly, if $f$ has a pole of order $m$ when $w=z$, then $f(w)=g(w) /(w-z)^{m}$ and $g(w) \neq 0$. Hence,

$$
\oint_{\gamma} \frac{f^{\prime}(w) h(w)}{f(w)}=\oint_{\gamma} \frac{-m h(w)}{w-z}=-m h(z)
$$

By combining (1), 2) and the lemma Cauchy_theorem_singularities 5 , we can show

$$
\oint_{\gamma} \frac{f^{\prime}(w) h(w)}{f(w)}=2 \pi i\left(\sum_{p \in \text { zeros }} n(\gamma, p) h(p) \operatorname{zo}(f, p)-\sum_{p \in \text { poles }} n(\gamma, p) h(p) \operatorname{po}(f, p)\right)
$$

where $\operatorname{zo}(f, p)$ (or $\operatorname{po}(f, p))$ is the order of zero (or pole) of $f$ at $p$, and the proof is now complete.

\subsection{Remarks}

Our definitions and lemmas in Section 4.1 roughly follow Stein and Shakarchi [16, with one major exception. When $f$ has a pole of order $n$ at $z$, Stein and Shakarchi define residue as

$$
\operatorname{Res}(f, z)=\lim _{w \rightarrow z} \frac{1}{(n-1) !} \frac{d^{n-1}}{d w^{n-1}}\left[(w-z)^{n} f(w)\right]
$$

while our lemma residue_porder states

$$
\operatorname{Res}(f, z)=\frac{1}{(n-1) !} \frac{d^{n-1}}{d w^{n-1}} h(z)
$$

where $f(w)=\frac{h(w)}{(w-z)^{n}}$ and $h(w)$ is holomorphic and non-vanishing near $z$. Note, $h(w)=(w-z)^{n} f(w)$ only when $w \neq z$, since $f(w)$ is a pole (i.e. undefined) when $w=z$. Introducing the function $h$ eliminates the technical difficulties of reasoning about limits formally.

\footnotetext{
${ }^{5}$ Either the lemma Cauchy_theorem_singularities or the lemma Residue_theorem suffices in this place.
} 


\section{Rouché's Theorem}

Given two functions $f$ and $g$ holomorphic on an open set containing a path $\gamma$, if

$$
|f(w)|>|g(w)|
$$

for all $w \in \gamma$, then Rouché's Theorem states that $f$ and $f+g$ have the same number of zeros counted with multiplicity and weighted with winding number:

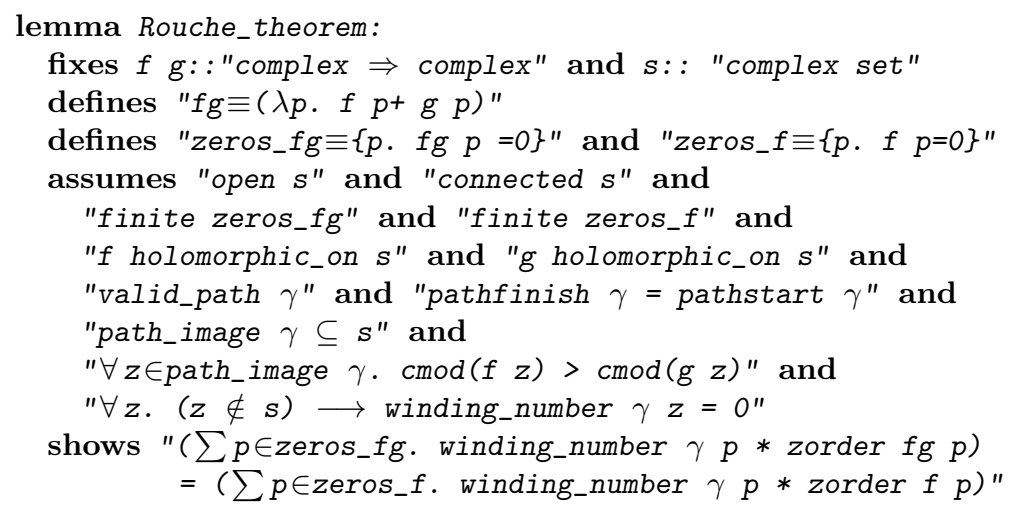

Proof. Let $\mathbb{Z}(f+g)$ and $\mathbb{Z}(f)$ be the number of zeros that $f+g$ and $f$ has respectively (counted with multiplicity and weighted with winding number). By the argument principle, we have

$$
\mathbb{Z}(f+g)=\frac{1}{2 \pi i} \oint_{\gamma} \frac{(f+g)^{\prime}}{f+g}=\frac{1}{2 \pi i} \oint_{\gamma} \frac{f^{\prime}}{f}+\frac{1}{2 \pi i} \oint_{\gamma} \frac{\left(1+\frac{g}{f}\right)^{\prime}}{1+\frac{g}{f}}
$$

and

$$
\mathbb{Z}(f)=\frac{1}{2 \pi i} \oint_{\gamma} \frac{f^{\prime}}{f}
$$

Hence, $\mathbb{Z}(f+g)=\mathbb{Z}(f)$ holds if we manage to show

$$
\frac{1}{2 \pi i} \oint_{\gamma} \frac{\left(1+\frac{g}{f}\right)^{\prime}}{1+\frac{g}{f}}=0
$$

As illustrated in Fig. 4, let

$$
h(w)=1+\frac{g(w)}{f(w)}
$$

Then the image of $h \circ \gamma$ is located within the disc of radius 1 centred at 1 , since $|f(w)|>|g(w)|$ for all $w$ on the image of $\gamma$. In this case, it can be observed that 0 lies outside $h \circ \gamma$, which leads to

$$
\oint_{h \circ \gamma} \frac{d w}{w}=n(h \circ \gamma, 0)=0
$$


where $n(h \circ \gamma, 0)$ is the winding number of $h \circ \gamma$ at 0 . Hence, we have

$$
\oint_{\gamma} \frac{\left(1+\frac{g}{f}\right)^{\prime}}{1+\frac{g}{f}}=\int_{0}^{1} \frac{h^{\prime}(\gamma(t))}{h(\gamma(t))} \gamma^{\prime}(t) d t=\int_{0}^{1} \frac{(h \circ \gamma)^{\prime}(t)}{(h \circ \gamma)(t)} d t=\oint_{h \circ \gamma} \frac{d w}{w}=0
$$

which concludes the proof.

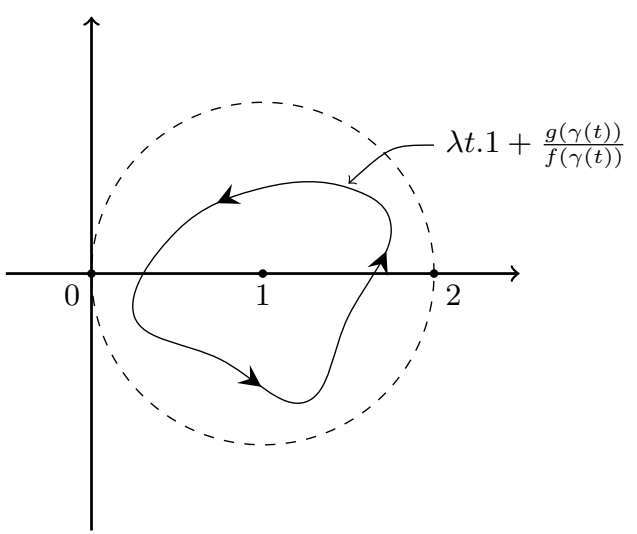

Fig. 4. The path image of $\lambda t .1+\frac{g(\gamma(t))}{f(\gamma(t))}$ when $|f(w)|>|g(w)|$ for all $w$ on the image of $\gamma$

Our proof of the lemma Rouche_theorem follows informal textbook proofs [3, 15, but our formulation is more general: we do not require $\gamma$ to be a regular closed path (i.e. where $n(\gamma, w)=0 \vee n(\gamma, w)=1$ for every complex number $w$ that does not lie on the image of $\gamma$ ).

\section{Related Work}

HOL Light has a comprehensive library of complex analysis, on top of which the prime number theorem, the Kepler conjecture and other impressive results have been formalized [11, 12, 13, 14. A substantial portion of this library has been ported to Isabelle/HOL. It should be not hard to port our results to HOL Light.

Brunel [6] has described some non-constructive complex analysis in Coq, including a formalization of winding numbers. Also, there are other Coq libraries (mainly about real analysis), such as Coquelicot [5] and C-Corn [10. However, as far as we know, Cauchy's integral theorem (which is the starting point of Cauchy's residue theorem) is not available in Coq yet.

\section{Conclusion}

We have described our formalization of Cauchy's residue theorem as well as two of its corollaries: the argument principle and Rouché's theorem. The proofs are 
drawn from multiple sources, but we were still obliged to devise some original proofs to fill the gaps. We hope our work will facilitate further work in formalizing complex analysis.

Acknowledgements. We are grateful to John Harrison for his insightful suggestions about mathematical formalization, and also to the anonymous reviewers for their useful comments on the first version of this paper. The first author was funded by the China Scholarship Council, via the CSC Cambridge Scholarship programme.

\section{References}

[1] Ahlfors, L.V.: Complex Analysis: An Introduction to the Theory of Analytic Funtions of One Complex Variable. McGraw-Hill, New York (1966)

[2] Avigad, J., Hölzl, J., Serafin, L.: A formally verified proof of the central limit theorem. CoRR abs/1405.7012 (2014)

[3] Bak, J., Newman, D.: Complex Analysis. Springer (2010)

[4] Basu, S., Pollack, R., Roy, M.F.: Algorithms in Real Algebraic Geometry. Springer (2006)

[5] Boldo, S., Lelay, C., Melquiond, G.: Coquelicot: A user-friendly library of real analysis for Coq. Mathematics in Computer Science 9(1), 41-62 (2014)

[6] Brunel, A.: Non-constructive complex analysis in Coq. In: 18th International Workshop on Types for Proofs and Programs, TYPES 2011, September 8-11, 2011, Bergen, Norway. pp. 1-15 (2011)

[7] Bruno Brosowski, F.D.: An elementary proof of the Stone-Weierstrass theorem. Proceedings of the American Mathematical Society 81(1), 89-92 (1981)

[8] Caviness, B., Johnson, J.: Quantifier Elimination and Cylindrical Algebraic Decomposition. Springer (2012)

[9] Conway, J.B.: Functions of One Complex Variable, vol. 11. Springer, New York, second edn. (1978)

[10] Cruz-Filipe, L., Geuvers, H., Wiedijk, F.: C-CoRN, the constructive Coq repository at Nijmegen. In: Mathematical Knowledge Management. pp. 88-103. Springer (2004)

[11] Hales, T.C., Adams, M., Bauer, G., Dang, D.T., Harrison, J., Hoang, T.L., Kaliszyk, C., Magron, V., McLaughlin, S., Nguyen, T.T., Nguyen, T.Q., Nipkow, T., Obua, S., Pleso, J., Rute, J., Solovyev, A., Ta, A.H.T., Tran, T.N., Trieu, D.T., Urban, J., Vu, K.K., Zumkeller, R.: A formal proof of the Kepler conjecture. arXiv $1501.02155(2015)$

[12] Harrison, J.: Formalizing basic complex analysis. In: Matuszewski, R., Zalewska, A. (eds.) From Insight to Proof: Festschrift in Honour of Andrzej Trybulec. vol. 10(23), pp. 151-165. University of Białystok (2007)

[13] Harrison, J.: Formalizing an analytic proof of the Prime Number Theorem (dedicated to Mike Gordon on the occasion of his 60 th birthday). Journal of Automated Reasoning 43, 243-261 (2009)

[14] Harrison, J.: The HOL Light theory of Euclidean space. Journal of Automated Reasoning 50, 173-190 (2013)

[15] Lang, S.: Complex Analysis. Springer (1993)

[16] Stein, E.M., Shakarchi, R.: Complex Analysis, vol. 2. Princeton University Press (2010) 